\title{
TRATAMIENTO DE LA PARÁLISIS FACIAL
}

\author{
José PEREYra KäFer *
}

La terapéutica de la parálisis facial puede ser encarada desde varios puntos de vista. Desde el punto de vista de las causas que han determinado la parálisis facial serán el neurólogo (parálisis centrales, parálisis de Bell), el neurocirujano (neurinomas del acústico, meningiomas, fracturas de la base), el cirujano general (fracturas de la base, tumores parotídeos), el otólogo (otitis crónicas, operaciones radicales de la mastoides) los llamados a intervenir primordialmente, pero es posible que en el curso evolutivo de la enfermedad más de uno de los respectivos especialistas tengan que resolver la conducta a seguir o encargarse del tratamiento. De ahi el amplio interés del tema.

Personalmente, he creído que debía encarar el tema desde el punto de vista neurológico y en base, no sólo del conocimiento de la literatura sobre el asunto, sino muy particu!armente de mi personal experiencia de neurólogo clínico.

\section{BASES DEL TRATAMIENTO}

El tratamiento de la parálisis facial tiene, razonablemente, que basarse en: a) tipo de la parálisis; b) etiología y naturaleza de la lesión causal; c) lugar en el cual se ha producido la lesión; d) intensidade o magnitud de la lesión y de la parálisis; e) tiempo transcurrido entre la iniciación de la parálisis y el examen del enfermo.

Si bien en el caso de la parálisis por reblandecimiento encefálico como en el sindromo de Millard-Gubler o en una compresión o destrucción de la vía opérculonuclear por un tumor, una hemorragia o un reblandecimiento, con parálisis de los miembros homolaterales, o en el caso de una sección quirúrgica en el curso de la extirpación de una neoformación parotídea, no puede haber mayor disparidad de opiniones sobre la causa y la naturaleza de la lesión, no ocurre lo mismo en numerosos casos de parálisis periféricas y en buena parte el tratamiento de estas últimas estará condicionado por la concepción etio y fisiopatogénica que tengamos al respecto.

Relato oficial al XIX Congreso Internacional de Oto-neuro-oftalmología (São Paulo, Brasil; junio de 1954).

* Profesor Adjunto de Clínica Neurológica en la Facultad de Ciencias Médicas de Buenos Aires. Jefe del Servicio de Clínica Neurológica del Policlínico "Ramos Mejía" de Buenos Aires. 
No poderios, sin embargo, entrar a considerar estos importantes aspectos, pues son motivo de otro relato. No obstante debemos distinguir los diferentes tipos para circunscribir el tema y guardar cierto orden.

Debemos diferenciar una parálisis central, córtico-nuclear y otra por lesión periférica, núcleo-muscular o de la unidad motora. En el primer tipo la conducta terapéutica estará regida por la naturaleza y ubicación de la lesión: trauma craneano, compresión o infiltración tumoral, encefalitis, reblandecimiento o hemorragia encefálica. En un primer período el tratamiento será el de la afección causal, ulteriormente será el común de las hemiplegías o hemiparesias (masajes para mantener el buen estado circulatorio y trófico de los músculos, reeducación de los movimientos mediante ejercicios controlados por el enfermo frente al espejo, cirugía estética).

Én el segundo tipo o núcleo-muscular la lesión puede estar en: 1) el núcleo del facial; 2) sus fibras radiculares dentro del puente; 3) el espacio subaracnóideo; 4) el conducto auditivo interno; 5) el acueducto de Fallopio; 6) la cara.

En el primer caso, constituido casi exclusivamente por la poliomielitis anterior itguda y algunas neuronitis, también el tratamiento será el de la enfermedad causal y además deberán agregarse desde un principio los mismos procedimientos terapéuticos generales que se emplean en la parálisis de Bell para mantener los músculos en buenos condiciones.

'I'ambién en la parálisis facial pontina (hemiplegías alternas de Millard-Gubler) debidas a accidentes vasculares, desde un principio y siempre que las condiciones generales del enfermo lo permitan, efectuaremos el tratamiento de los músculos como en la parálisis de Bell. Los sucesivos exámenes clínicos e instrumentales nos dirán si existen posibilidades de recuperación, y por lo tanto, si conviene insistir en este tipo de tratamiento o si la extensión e importancia de la lesión es tal que toda recuperación funcional será imposible, en cuyo caso, si hubiera lugar, deberá procederse ulteriormente al empleo de las medidas correctoras propias de la cirugía plástica. Aquí puede ser de importancia la tarsorrafia precoz para evitar la ulceración corneana.

En el caso de lesiones en el espacio subaracnóideo debemos considırar particularmente las parálisis faciales del período secundario de la sífilis, las producidas por la meningitis gomosa de la base, las de la meningitis tuberculosa, las de los tumores y las aracnoiditis, etc. Aquí también lo más importante es el tratamiento de la causa y ulteriormente, si persiste el déficit motor, se aplicarán los mismos procedimientos que veremos más adelante para las formas de Bell, particuiarmente lo que se refiere a la fisioterapia muscular y a los cuidados ortopédicos.

En el conducto auditivo interno debemos tener sobre todo en cuenta los tumores del octavo par y los traumatismos de la base del cráneo. Aquí también lo fundamental será tratar la causa. En el caso particular de los traumas de la base puede efectuarse la recuperación espontánea (sección parcial, compresión por hematoma, escasa separación de los cabos proximal y distal), lo cual se comprobará por el estudio electrico o bien será necesaria la anastómosis. A penas el estado del paciente lo permita, deberá efectuarse el tratamiento de los músculos como luego indicaremos y si los exámenes frecuentemente realizados señalan la acentuación de la degeneración, deberá procederse quirúrgicamente.

En el canal de Fallopio excepcionalmente podrá lesionarse el facial por una hemorragia en un hipertenso arterial como comprobáron anatómicamente Moxon, Romberg, y Monier-Vinard y Puech 24, teniendo nosotros solamente un caso de observación personal que creemos seguro. Si así ocurre el tratamiento será similar al de la parálisis de Bell con las lógicas variantes impuestas por su particular etiología. En este acueducto además el facial podrá ser lesionado en el curso de operaciones óticas o espontaneamente clurante la evolución de enfermedades 
propias de esta región y tan familiares para el otólogo, y por traumatismos de la base del cráneo. El examen directo del nervio o la evolución, según los casos, indicarán si deberá procederse a una sutura término-terminal o efectuarse un injerto o proceder a liberar al nervio de la compresión de los tejidos que lo comprimen sin abrir su vaina para evitar que el proceso infeccioso se extienda al nervio y ocasione su fibrosis, lo cual obligaría a la resección y al injerto (Collier). Én el canal de Fallopio la afección más común es la parálisis de Bel, comunmente llamada también "a frigore" (parálisis de Bell aguda monosintonática de origen "reumático" o criptogenético, según Kinnier Wilson ${ }^{22}$ ).

Én el cuello y la cara el facial es lesionado por heridas por arma blanca o de fuego, tumoraciones, intervenciones quirúrgicas en el curso de extirpación de neoplasias. Suturas, injertos y operaciones plásticas se efectuarán según la extensión, naturaleza y etapa evolutiva de la parálisis.

A continuación nos ocuparemos particularmente del tratamiento de la parálisis de Bell, cuyas directivas generales, como ya hemos mencionado reiteiradamente, son de aplicación a los otros tipos de parálisis faciales.

\section{TRATAMIENTO DE LA PARALISIS DE BELL}

Es un hecho evidente que la terapia de la parálisis facial no puede encomendarse a ayudantes prácticos sin contralor médico especializado directo. Es el médico conocedor de fisiopatología y clínica neuromuscular quien debe orientar y de ser posible, efetuar el tratamiento. Y éste será guiado y modificado de acuerdo a reiterados exámenes clínicos e instrumentales, los cuales permitirán, además, establecer el pronóstico en las distintas etapas evolutivas de la recuperación. A tal efecto conviene tener presentes las consideraciones que van a continuación.

La clasificación de Erb, basada en el electrodiagnóstico, sigue siendo útil en líneas generales para fijar el pronóstico y guiar el tratamiento. Considera tres formas de gravedad progresiva. En la forma leve, sin trastornos de la excitabilidad, la curación se obtiene dentro de los veinte días aproximadamente. En la torma mediana hay reacción de degeneración (RD) parcial y la parálisis cura entre uno y dos meses. En la forma grave se comprueba la RD completa; la parálisis puede curar pero en ocasiones no retrocede o cura con contractura.

Esste esquema es, evidentemente, demasiado rígido, pero sus inconvenientes se obvian en general si, en vez de dar un valor absoluto al electrodiagnóstico efectuado al final de la segunda o tercera semana, se practican reiterados exámenes eléctricos, ya que, sobre todo una RD parcial puede hacerse ulteriormente completa por persistencia o acentuación de la causa que generó la parálisis. Además todos sabemos que no hay un exacto paralelismo entre la evolución clínica y las reacciones eléctricas. Así es muy común que casos con RD parcial tarden mesés en curar, o que acentuadas recuperaciones clínicas sigan acompañándose durante largo tiempo de reacción de Remak. Cabe citar aquí las palabras de Bauwens 5 pronunciadas en un symposium sobre tratamiento de la parálisis facial en 1950: "Si bien este esquema ha provocado alguna crítica debido a su inseguridad en las manos de posteriores autoridades, debe notarse que éstas, raramente, si es que alguna vez lo han hecho, han contribuído con algo de valor para suplementar o proponer un esquema infalible como sustituto".

James y Russel 2i hacen una clasificación, de acuerdo al curso evolutivo, que nos parece interesante. Ellos dividen a las parálisis de Bell en dos grupos. En 
el primero, que comprende alrrededor del $80 \%$ de los casos, no hay $\mathrm{RD}$; la recuperación comienza entre una a tres semanas y se completa en cuatro a acho; cste curso clínico indicaría un bloque parcial del nervio, muy semejante a una parálisis por compresión. En el segundo grupo se comprueba RD y los músculos permanecen dos o más meses completamente paralizados. El nervio estaría muy lesionado y la recuperación sólo podría hacerse por regeneración, es decir por reinervación muscular, tardando de tres a nueve meses desde el comienzo. En estos casos de regeneración las sincinesias son una consecuencia casi obligada y por lo tanto la recuperación es incompleta ya que se producen ramificaciones y desviaciones de los axones regenerados.

Por último, la electromiografía nos demostrará si hay unidades motoras totalmente denervadas (potenciales de fibrilación), si algunas unidades motoras responden a la voluntad o a los estímulos farádicos aplicados al tronco del nervio $y$, en el período de recuperación, registros caracteríscicos podrán obtenerse antes de aparecer los movimientos voluntarios, lo cual confiere a este método una enorme importancia.

Como acabamos de ver, un alto porcentaje de parálisis faciales se curan espontáneamente y en estos casos la regresión es, en general, bastante importante en las primeras dos a tres semanas pero, para el caso individual, no existe ningún signo de valor absoluto que permita establecer con certeza el pronóstico alejado, por lo cual todos los casos deben ser sometidos al tratamiento precoz.

Hasta no hacen muchos años era corriente la idea de que debía esperarse unas dos a tres semanas antes de iniciar el tratamiento activo y que la electricidad así como cualquier otro método de estimulación muscular eran peligrosos durante ese lapso de tiempo pues podrian favorecer la aparición de la contractura, los movimientos masivos y los espasmos de los músculos faciales. Pero existe ya suficiente evidencia de que tal actitud es errónea y que sólo beneficios pueden obtenerse del tratamiento precoz bien conducido. Como dice Bierman ${ }^{6}$, "el viejo criterio de esperar diez o más días después del comienzo de la parálisis es indefendible".

El tratamiento debe tener por finalidades corregir: a) la desfiguración del rostro debida a la pérdida del tono muscular permanente por ruptura del reflejo miotático, con relajación cutánea y a la tracción de los músculos del lado sano (facial inferior); b) las algias, muchas veces presentes en el comienzo de la enfermedad y menos frecuentemente persistentes; c) los trastornos funcionales dependientes de la parálisis, en particular del orbicular de los párpados, del orbicular de los labios, zigomáticos y bucinador, con las consiguientes perturbaciones de la oclusión palpebral, la correcta circulación de las lágrimas, la palabra y la alimentación; d) los trastornos psíquicos reactivos, debidos a la desfiguración del rostro en reposo y en particular durante la ejecución de movimientos voluntarios y automáticos (palabra, risa, etc.).

Para cumplir con estas finalidades el tratamiento debe realizarse procurando actuar: a) sobre el nervio facial; b) sobre los músculos para- 
lizados; c) sobre la piel del lado afectado de la cara; d) sobre el estado psíquico del paciente.

Encaramos el tratamiento de la parálisis de Bell suponiendo que la misma se debe a una afección del tronco nervioso dentro del acueducto de Fallopio, que el exiguo calibre del mismo es extraordinariamente apto para que el tronco nervioso sea fácilmente comprimido por congestión, edema o infiltración perinerviosa y nerviosa y que esta compresión origina rápidamente hipo o anoxia del nervio al actuar sobre los vasa nervorum.

Por lo que antecede consideramos que en un principio deben emplearse los elementos terapéuticos que pueden tener acción anticongestiva o antiflogística, disminuyendo o suprimiendo la compresión y la isquemia. En este sentido, desde hace ya muchos años utilizamos la radioterapia profunda y el calor local y últimamente el ACTH o córticotropina. Con el mismo criterio Bauwens dice que durante las primeras etapas de la parálisis cualquier cosa debe suministrarse para aliviar la presión y congestión y resolver la inflamación.

La parálisis de Bell sobreviene unas veces sin pródromos, pero otras, durante uno a varios días, los pacientes acusan dolores más o menos intensos en la región mastoidea, el oído, el lado correspondiente del cuello y ocasionalmente el hombro homolateral. Excepcionalmente toda la hemicara es dolorosa espontáneamente y a la presión. Estos dolores habitualmente desaparecen poco después de presentarse la parálisis o persisten algunos días. Sólo en alguna ocasión los hemos visto durar seis meses a pesar de los analgésicos que por prescripción médica había tomado la enferma y ceder luego rápidamente a la radioterapia que indicamos apenas vimos a la paciente por primera vez. Si bien para calmar los dolores pueden utilizarse los comunes analgésicos (aspirina, antipirina, piramidón, etc.), nosotros aconsejamos la radioterapia profunda sobre la región correspondiente al acueducto de Fallopio pues a su acción analgésica se agrega la antiflogística, que creemos más importante aún y que ya hemos mencionado. Si el dolor se extiende a toda la hemicara y los tejidos son hipersensibles a la presión, también esta zona debe ser irradiada. En la primera sesión se emplean 50 unidades $\mathrm{r}$, y en las sucesivas, de 50 á 90; las aplicaciones deben efectuarse cada 2 á 3 días, siendo suficientes de 3 á 6 sesiones.

En cuanto al calor, puede ser utilizado en forma muy variada desde las cataplasmas comunes, la bolsa con agua caliente, el fomento eléctrico, los rayos infrarrojos, las ondas cortas. Nosotros preferimos a estas últimas, que indicamos desde el primer día, en sesiones diarias de unos 10 minutos de duración, sobre la hemicara incluyendo la región del acueducto de Fallopio.

El curso, como ya dijimos, es habitualmente benigno, pero como ello no puede preverse anticipadamente consideramos que estos procedimientos 
deben emplearse en forma sistemática. Recién después de unos 15 a 20 dias, por la evolución clínica y, si es necesario, por los exámenes eléctricos, podremos prever aproximadamente el curso futuro y saber con que procedimiento debemos continuar.

Con la misma finalidad de actuar sobre el probable mecanismo que origine la parálisis de Bell y teniendo en cuenta que "la cortisona se ha mostrado en muchos casos capaz de aliviar ciertos trastornos congestivos inespecíficos", Rothendler ${ }^{29 a}$, en 1951, utilizó en un caso de intensa parálisis facial el tratamiento a las 24 horas del comienzo con $100 \mathrm{mgr}$. de cortisona cada 6 horas; la mejoría comenzó a las 48 horas; al quinto día redujo la dosis a $100 \mathrm{mgr}$. cada 8 horas, al séptimo a 100 cada 24 horas, continuando durante seis días más; a los 13 días el paciente estaba totalmente curado. Robbins ${ }^{28}$, en 1952 , relató otro caso, con ageusia de los dos tercios anteriores de la lengua, cuyo tratamiento se efectuó por vía oral con $500 \mathrm{mgr}$. diarios rèpartidos en cinco dosis de $100 \mathrm{mgr}$. cada una, iniciándose al noveno día de producida la parálisis; dice el autor que al finalizar el primer día había una notable mejoría, que al segundo el gusto ya era normal y que curó en unos doce días. Ultimamente (1953) Rothendler ${ }^{29 a}$ insistió nuevamente sobre el empleo precoz de la cortisona. Nosotros en los últimos tiempos hemos utilizado el ACTH y, si bien nuestra experiencia es muy escasa, creemos que, tanto la cortisona como la corticotropina merecen realmente ser ensayadas con más amplitud, especialmente si se tienen en cuenta los resultados que se logran en otros proresos en los cuales la congestión y el edema parecen jugar un importante papel patogénico, como en la esclerosis múltiple y el sindrome de Guillain-Barré.

La radioterapia, las ondas cortas, la cortisona y el ACTH tienden a actuar sobre el nervio desde el comienzo de la enfermedad y sus posibilidades terapéuticas son tanto mayores cuanto más precozmente se $\mathrm{km}$ plean, sin esperar a saber si la parálisis facial será grave o no. Este criterio - acción precoz sobre el nervio - que sostenemos desde hace casi treinta años, está de acuerdo con lo que, con buen juicio, dicen James y Russell "21 "Lo que nosotros requerimos por lo tanto es alguna forma activa de tratamiento que pueda suministrarse desde el primer día de la parálisis y que permitirá al nervio facial evitar la degeneración walleriana y sus perturbadoras consecuencias".

Si se acepta con Duel ${ }^{13}$ que la parálisis de Bell se debe a congeslión e inflamación del nervio en la porción ósea compacta del acueducto de Fallopio, es decir en la porción del canal que se extiende entre el conducto horizontal del laberinto y el agujero estilomastóideo - y se tienen en cuenta las comprobaciones experimentales de Lewis, Pickering y Rothschild de que las parálisis periféricas por compresión se deben a la isquemia del segmento comprimido, surge la necesidad de liberar inmediatamente a las fibras nerviosas de esa compresión. Es lo que noso!ros 
hemos intentado con la radioterapia y las ondas cortas y la corticotropina y Rothendler con la cortisona.

Pero otros han intentado ser más ejecutivos (Duel, Morris, Tickle, Kettel) realizando operaciones decompresivas, abriendo el acueducto $\mathrm{y}$ seccionando la vaina del nervio siguiendo la vía iniciada por Duel y Ballance en los casos de lesión del VII par en el curso de las mastoidectomías. Morris ${ }^{26 a}$ dice que, en la parálisis de Bell, "debido a la incapacidad del nervio y su vaina para expandirse como resultado de la congestión e inflamación, las fibras nerviosas están comprimidas en el canal óseo y cortados los impulsos nerviosos. Desprovistas de su aporte sanguíneo las fibras nerviosas mueren desde el punto de la compresión havia la periferia pero las vainas persisten. Si entonces el nervio es expuesto y la principal vaina más externa incindida, se restablecerá la circulación y eventualmente las fibras nerviosas alcanzarán sus respectivas vainas más abajo y se restablecerá la función". Considera que, si al final de la cuarta semana no hay respuesta farádica se debe exponer el nervio e incindir la vaina. Si existe mejoría pero ésta se detiene y todavía hay mucha desfiguración, epífora, etc., aconseja operar igualmente y aún practicar un injerto: lo cual no interferiría con las fibras ya recuperadas.

En un nuevo trabajo ${ }^{26 b}$, Morris dice que cuanto más precozmente se realiza la intervención decompresiva menos peligro habrá de permanente detcrioración de la función muscular pero reconoce que la elección de los casos para operar no es fácil. No obstante, considera que si la recuperación con los tratamientos usuales de estimulación muscular no es satisfactoria, al cabo de seis semanas a dos meses se debe operar. Relata Morris un caso con muy incompleta recuperación al cabo de un año y que permaneció estacionario durante otros siete años y que se benefició francamente con la decompresión, como para mostrar a las cuatro semanas de la misma una neta mejoría. Es sin duda difícil comentar este resultado.

Mucho se ha escrito sobre este tipo de intervención y sería ocioso resumir toda la literatura al respecto. Mencionaremos sólo algunas opiniones que nos parecen serias y bien fundadas. James y Russell ${ }^{21}$ creen que este procedimiento es irracional porque si la decompresión es realmente útil debería ser efectuada dentro de las dos semanas del comienzo, cuando se presenta la evidencia eléctrica de la degeneración nerviosa, como aconsejaron Ballance y Duel ${ }^{4}$ en 1932. La operación efectuada de seis a ocho semanas del comienzo coincide con el principio de la regeneración normal y el cirujano puede atribuirse falsamente el mérito de muchos casos de regeneración espontánea a los tres o cuatro meses. Además, como toda regeneración facial, no impediría las sincinesias.

Callier ${ }^{9 b}$, en 1950, realizó un estudio muy completo. Entre otras cosas, la autora señala que debe recordarse la posibilidad del origen nuclear de algunas parálisis faciales por aquellos inclinados a recomendar 
la operación decompresiva. Considera que no es posible obtener ninguna evidencia clara de la anatomía patológica de los hallazgos encontrados en ocasión de las decompresiones. Recuerda que si se toma la inexcitabilidad farádica como testimonio de degeneración total, la recuperación tendrá que hacerse por regeneración, lo cual tomará tiempo y en todos los casos se asociará con los defectos propios de la reinervación, es decir sincinesias y paresia, y que la creencia de que la decompresión efectuada de ocho a diez semanas después del comienzo de la parálisis ha sido responsable de la recuperación en pocos días no toma en cuenta el tiempo que tarda en producirse la regeneración. Por otra parte, desde que pudo contar la ayuda de la electromiografía, no halló ningún caso que justificase la decompresión quirúrgica.

Como sucede en todos los casos de parálisis, a la perturbación inicial, nerviosa, siguen alteraciones musculares, cutáneas, vasculares, etc., variables según el tipo de parálisis y la región corporal afectada. En los músculos tiene lugar un proceso de atrofia y fibrosis y además ellos sufren las consecuencias de la acción de los núsculos sanos, que los traccionan, agregándose además la acción de la gravedad, todo lo cual produce el estiramiento muscular.

Como es sabido, para obtener una buena recuperación funcional no basta la regeneración del nervio; es necesario que los axones encuentren músculos funcionalmente aptos y no bandas musculares atróficas y distendidas o sólo cintas fibrosas, en cuyo caso toda recuperación funcional es imposible.

Para actuar sobre los músculos y eil parte indirectamente sobre la piel, debemos emplear, siguiendo aproximadamente a Bierman, la inmovilización, el calor, la estimulación eléctrica, el masaje, y los ejercicios activos.

Inmovilización - Las alteraciones musculares por estiramiento, clínica y anátomicamente conocidas y universalmente aceptadas, han sido además experimentalmente demostradas por Eisenhauer y Key ${ }^{11}$, quienes comprobaron que la atrofia por falta de uso era más severa en los músculos estirados que en los que se mantenían relajados o en un estado intermedio.

Pickerill y Pickerill ${ }^{27}$ asignan gran importancia al estiramiento inmediato y continuado de los músculos paralizados por los del lado sano de la cara y dicen que en los casos de sección del facial producida durante la extirpación quirúrgica de la parótida, si se evita el estiramiento, el tono de los músculos paralyzados disminuye muy poco en el curso de las tres semanas que transcurren hasta que se efectúa la cirugía plástica, en contraste con lo que ocurre cuando no se ha evitado el estiramiento precoz.

Por esto es útil indicar al paciente con parálisis de Bell que efectúe el mínimo de gesticulaciones faciales, que hable poco y entre dientes y 
que trate por todos los medios de controlar su mímica facial, manteniendo, dentro de lo posible, su cara inmóvil. Al respecto James y Russell ${ }^{21}$ recuerdan que ya en 1919 Spiller $^{31}$ recomendó que se debía procurar una expresión facial plácida y relajada.

Desde el primer día y mientras los dolores no lo impidan, deben emplearse medios de contención destinados a evitar el estiramiento muscular y de la piel, particularmente en las personas de edad. A tal efecto se utilizan para el párpado inferior, siguiendo a Bierman ${ }^{6}$, dos tiras de esparadrapo de unos 6 a $7 \mathrm{~mm}$. de ancho por unos $10 \mathrm{~cm}$. de largo, que se cruzan entre sí por debajo del párpado inferior, naciendo a unos $2 \mathrm{~cm}$. por dentro y por fuera de la parte media de la órbita y se dirigen oblicuamente hacia arriba, respectivamente hacia afuera y hacia adentro, para terminar en la parte externa de la frente, cerca de la línea capilar una y la otra, después de cruzar la raíz de la nariz, en la piel de la frente del lado opuesto. Previamente a la fijación el párpado inferior y la parte superior de la mejila son sostenidos hacia arriba en buena posición, especialmente antes de adherir el esparadrapo a la frente. Teniendo en cuenta que, durante el decúbito horizontal, la tracción debida a la acción de la gravedad es mucho menor, estas tiras de esparadrapo pueden ser removidas durante la noche.

Para mantener en adecuada posición la parte inferior de la cara y evitar el estiramiento de los músculos paralizados por la acción de los del lado sano, los Pickerill ${ }^{2 i}$ aconsejan la colocación de dos bandas de esparadrapo de unos 10 a $15 \mathrm{~mm}$. de ancho, que partiendo desde la parte inferior de la mejilla del lado sano y pasando sobre la piel de los labios superior e inferior, cruzan la mejilla del lado enfermo sin adherir a ella por cuanto previamente la piel de ésta ha sido suavemente untada con vaselina $o$ aceite; traccionando $t$ l lado sano, son adheridas fuertemente sôbre la piel de la apófisis mastoides del lado enfermo. Son varios los autores que consideran que este procedimiento no se tolera y prefieren el dispositivo de alambre que se engancha en la comisura de los labios del lado paralizado y la sostiene en buena posición, fijándose por el otro extremo en la oreja homolateral como una patilla de anteojo. Pero, apesar de que Wilson considera que este dispositivo puede en gran parte evitar la ulterior desfiguración, consideramos al de Pickerill fisiológicamente útil, contrariamente al gancho de alarnbre, pues aquel impide la tracción que en estos casos realizan los músculos del lado sano (zigomáticos, buccinador, orbicular de los labios, etc.), mientras que el gancho de alambre deja libre a estos músculos y fija la comisura del lado enfermo exagerando los trastornos que pretende corregir. Probablemente la férula intraoral ideada por Allen y Northfield ${ }^{1}$ y de la cual no tenemos experiencia personal, sea preferible al gancho. Bierman ${ }^{6}$ reconoce que es mejor y cosméticamente superior pero tampoco impide la tracción del lado sano. 
Calor - Consideramos de importancia la aplicación de calor para actuar, en un principio y como ya dijimos, sobre el nervio y los músculos y ulteriormente sobre los músculos. Como dice Bierman ${ }^{6}$, el calor favorece la contracción muscular y al respecto trae a colación el hecho de que, si un paciente llega desde un lugar frío, es posible que no se le pueda descubrir ningún movimiento mientras que después de la aplicación de calor estos pueden ser fácilmente comprobables. Además, Asmussen y Boje demostraron que un músculo funciona mejor después de calentado. En los músculos denervados observaron Bowden y Gutmann dilatación vascular y diapedesis hemática y leucocitaria. Como dice Bierman, el efecto hiperhemiante del calor puede contribuir a reducir este éstasis vascular.

Wilson ${ }^{22}$ recomienda la aplicación inmediata de calor local alrrededor del oído y del agujero estilimastóideo, seguramente en un intento de actuar sobre el nervio enfermo, cualquiera sea el método empleado y durante una semana más o menos; despreocupándose por lo tanto de su acción sobre los músculos, lo cual consideramos erróneo.

Es certo que todas las formas de calor pueden emplearse pero nosotros consideramos más útiles, sobre todo en un principio, para actuaren en profundidad sobre la vaina del nervio y los músculos, las ondas cortas que, como ya mencionáramos, indicamos desde el primer día en sesiones diarias. También desde el comienzo del tratamiento es útil prescribir, al mismo tiempo que las ondas cortas, aplicaciones más simples de calor local para actuar sobre los músculos y la piel (fomentación eléctrica, fomentos comunes de agua caliente, bolsa de goma con agua caliente, etc.), que pueden efectuarse dos a tres veces por día. Tienen la ventaja de poder ser hechas por el mismo paciente y por otra parte tienden a satisfacer su necesidad de un tratamiento activo. Las aplicaciones de ondas cortas no las prolongamos más alla de las tres a cuatro primeras semanas; en cambio, las otras formas de calor deben utilizarse mientras la involución de la parálisis no se haya detenido.

Electroterapia - Consideramos que debe efectuarse lo más precozmente posible. En el mismo sentido opinan numerosos autores y nosotros, personalmente no estamos de acuerdo con aquelos ${ }^{9}$ que consideran que "el tratamiento para limitar la atrofia y el estiramiento debe iniciar-se tan pronto como haya evidencia de degeneración del nervio". Esto recien puede apreciarse después de 15 a 20 días. Preferimos empezar el tratamiento apenas producida la parálisis, siempre que no haya contraindicaciones formales (algias intensas). Efectuamos habitualmente sesiones de galvanización al estado permanente, con electrodo positivo o indiferente en la región cérvico-dorsal y electrodo negativo o activo en forma de hemimáscara de Bergonié sobre el lado paralizado de la cara; sesiones de 15 a $30 \mathrm{mi}$ nutos de duración, con una intensidad de 10 a 20 Ma., día por medio. De este modo se obtiene una vasodilatación útil que activa los cambios inetabólicos y mejora la circulación y por lo tanto el trofismo muscular 
y cutáneo. Como dicen Delherm y Laquerrière ${ }^{11}$, en una región sometida a la corriente continua se observa la disminución de los edemas, de los éstasis circulatorios y Guilloz ha podido demostrar que durante su aplicación y durante cierto número de horas después de la misma aumenta la respiración elemental de la fibra muscular separada del organismo.

En general, después de la sesión de galvanoterapia al estado permanente efectuamos estimulaciones aisladas utilizando un mango excitador con una almohadilla de unos 5 a $7 \mathrm{~mm}$. de diámetro. Debe emplearse para cada músculo la mínima intensidad de corriente que produce una rontracción claramente visible, utilizar como polo activo aquel con el cual se obtiene la contracción con el mínimo amperaje o, como dicen Delherm y Laquerrière ${ }^{11}$, para cada músculo el polo que mejor lo excita y no exclusivamente el cátodo, como aconsejan Wilson ${ }^{22}$ y Bierman $^{6}$. Dejado El electrodo in situ, se efectúan unas 20 a 40 estimulaciones en cada músculo. Conviene, de ser posible, emplear un interruptor automático, pero lo mismo puede lograrse con un mango interruptor que habitualmente se maneja con la mano derecha mientras con la izquierda se regula la intensidad de la corriente. Las estimulaciones eléctricas espaciadas conviene efectuarlas diariamente y hasta dos veces por día ${ }^{22}$.

En las formas ligeras puede prescindirse de la sesión de galvanización al estado permanente y utilizar tan solo las contracciones aisladas. No debe, en cambio, emplearse este último procedimiento cuando para lograr la contracción muscular se requiere un elevado miliamperaje, por cuanto, entonces, la excitación difunde y hace contraer a otros músculos del mismo lado o del lado opuesto y la aplicación resulta dolorosa y desagradable para el enfermo, además de perjudicial. Con la mejoría la galvanización será gradualmente reducida o dirigida únicamente a los músculos que son tardíos en retomar su función ${ }^{22}$.

Gutmann y Gutmann ${ }^{17}$, que efectuaron un importante trabajo experimental en conejos, llegan a interesantes conclusiones que consideramos pertinente reproducir aquí: el ejercicio galvánico de los músculos denervados del conejo retarda y disminuye la atrofia muscular y acelera el retorno del músculo a su volumen inicial después de la reinervación; los músculos tratados muestran menos fibrosis y fibras musculares más gruesas con estriación más definida que los músculos no tratados; los músculos tratados muestran una mejor excitabilidad y contractilidad a la estimulación directa y acción refleja más vigorosa que los no tratados; cuanto más superficial es el músculo mejor mostrará el efecto del tratamiento. Para este trabajo experimental efectuaron tratamientos diarios en sesiones de 15 a 20 minutos, con estímulos de 4 a 6 Ma., produciendo de 500 a 600 contracciones con método bipolar.

También Hines ${ }^{18}$, trabajando con ratas blancas, comprobó que la estimulación eléctrica durante tres minutos diariamente retardaba apreciable- 
mente la atrofia muscular previa a la reinervación y aceleraba la recuperación del peso y la fuerza del músculo después de la reinervación.

Por último Jackson y Seddon ${ }^{20}$, en cincuenta y cuatro casos bien seguidos de parálisis cubital, aplicaron 90 estímulos a cada pequeño grupo muscular con intensidade suficiente para obtener una buena contracción, en series de 30 estímulos término medio por minuto, con cortos intervalos entre cada una de las tres series para prevenir la fatiga y ha!lando que este procedimiento empleado seis días semanalmente fue casi completamente efectivo para evitar la atrofia, excepto durante las semanas que siguen irmediatamente a la denervación, aún cuando durante este período el tratamiento redujo la velocidad de producción de la atrofia.

Para Collier ${ }^{9}$, el único valor de la galvanización consiste en mantener la contractilidad muscular, produciendo contracciones que, además, contribuirían a reducir, aunque no a suprimir, la fibrosis intersticial, lo cual ya sería bastante importante y Spillane ${ }^{30}$ considera que, si bien el exacto mecanismo de la atrofia no se conoce, hay actualmente buena evidencia de que puede ser reducida por la estimulación galvánica y que, si desde el comienzo se los hace contraer diariamente, los músculos decaen poco o nada.

En realidad, la estimulación galvánica es siempre útil al mantener en mejores condiciones el trofismo muscular, combatir el éstasis circulatorio, reducir la fibrosis y mejorar la contractibilidad. Como dice Spillane ${ }^{30}$, en un caso descuidado de parálisis de Bell es posible ver que la estimulación galvánica al principio no produce respuesta pero, después de más o menos una semana, los músculos pueden comenzar a contraerse y más adelante lo pueden hacer vigorosamente. Esto es más importante cuando hay degeneración y la curación debe depender de la regeneración del nervio y de la reinervación de los músculos.

Masajes - Los músculos faciales son sumamente delgados y por otra parte carecen de vainas o fascias superficiales que los aislen netamente del tejido celular subcutáneo. Adherentes a la piel y ubicados contra el plano óseo, son fácilmente accesibles a la acción del masaje supcrficial.

Por otra parte, la piel, privada del sostén de los músculos que en ella se insertan, se relaja y pende más o menos flácida según el estado trófico y la edad del enfermo, siendo esto mucho más manifiesto en las personas ancianas debido a la disminución del tejido elástico.

El masaje manual, efectuado por un kinesiólogo competente, tiene aquí las mismas indicaciones que en cualquier otra región corporal a los efectos de contribuir a mantener un buen estado trófico de los músculos. Pero, por las condiciones más arriba citadas, debe ser suave, con escasa presión, para no provocar la atricción de los músculos contra el plano óseo y efectuarse siguiendo líneas paralelas a los orificios natura'es de modo de es- 
timular la circulación cutánea y subcutánea y provocar contracciones mus. culares reflejas fásicas sin estirar la piel.

De ser posible conviene sea efectuado en sesiones diarias y, en caso contrario, por lo menos tres veces semanales, desde un principio, si no hay contraindicaciones y durante todo el tiempo que dure la retrocesión de la parálisis.

Ejercicios activos - Los consideramos de mucha importancia y deben efectuarse desde un principio si se trata de una paresia o cuando se inicia la recuperación en caso de parálisis. Como dise Wilson ${ }^{22}$, aún cuando sólo se obtenga al comienzo un mero temblor, el paciente debe practicar asiduamente y no conformarse en confiar pasivamente en lo que se hace por él sin su activa contribución personal. Bierman ${ }^{6}$ considera al ejercicio activo como la mejor forma de fisioterapia para músculos que funcionan pobremente.

Nosotros creemos que facilitan la restauración funcional al reducir la atrofia y la fibrosis y nunca hemos visto trastornos imputables a su práctica correcta.

Hines ${ }^{19}$, trabajando experimentalmente con los músculos gastrocnemios, mediante secciones de los nervios tibiales de ratas blancas adultas, comprobó que la inmovilización del músculo en posición neutral retarda la recuperación de la parálisis, perjudicando la restauración de la masa muscular y de la fuerza, que sigue a la reinervación, y que la actividad forzada inducida por la natación durante una hora diaria o ejercicio ea una jaula rotatoria durante dos horas, en ningún caso perjudicó a la regeneración neuromuscular. Llega a la conclusión de que el ejercicio facilita la recuperación de la parálisis debida a la lésion nerviosa periférica.

Nosotros ordenamos cuatro sesiones diarias de movimientos activos realizados frente al espejo. El paciente debe efectuar movimientos de oclusión palpebral, elevación de la ceja, desviación de la comisura bucal hacia el lado paralizado, oclusión labial, etc., evitando, en particular para el facial inferior, que se contraigan simultáneamente los músculos del lado sano provocando estiramiento. Aún para los músculos inervados por el facial superior, si es posible, conviene la contracción aislada en el lado paralizado para adquirir el dominio correcto de los mesmos. Por supuesto que, previamente, se habrá explicado al paciente porque no debe contraer los músculos del lado sano y cuales son los inconvenientes del estiramiento muscular. Estamos de acuerdo con Bierman ${ }^{6}$ cuando dice que, si es necesario, los músculos del lado sano deben ser contenido manualmente. También coincidimos en que hay posiciones más favorables que otras y que así, v.g. la oclusión palpebral puede ser más fácil en decúbito que en posición erecta, probablemente porque el orbicular no tiene que vencer la acción de la gravedad (párpado inferior).

La gran mayoría de los casos de parálisis facial se cura completamente o con tan pequeño déficit que resulta la curación estética y funcio- 
nalmente satisfactoria. Otros curan parcialmente, quedando algunos músculos total o casi totalmente denervados y terminando en total atrofia y Iiibrosis. Hay casos que llegan a nuestras manos después de mucho tiempo de producida la parálisis y nos encontramos con una reacción de degeneración absoluta, habiendo desaparecido prácticamente todo el tejido muscular subcutáneo de la cara. Pero también hemos visto otros, cuyo tratamiento había sido mal efectuado o abandonado casi desde un principio, con marcada deformación del rostro y RD parcial, a un año o dos del comienzo de la parálisis, reaccionar rápidamente con los ejercicios activos y la estimulación galvánica intermitente.

En los casos en que se necesita la regeneración del nervio es muy difícil que los resultados sean perfectos ${ }^{30}$. Contracturas, espasmos, sincinesias serán consecuencias habituales. Generalmente a los ocho a doce meses los resultados de la reinervación son definitivos pero es evidente, como dice Spillane: ${ }^{30}$, que puede pasar aproximadamente un año antes de que los movimientos faciales comiencen a retornar y la recuperación ser completamente satisfactoria a los dos años del comienzo de la parálisis.

¿Hasta cuando debe continuarse la fisioterapia? Si durante un plazo de dos a tres meses no se aprecia, clínica ni eléctricamente, ninguna recuperación adicional en un caso que mejoraba, podemos considerar que la mejoría ya no seguirá prosperando. Si ésta ha sido muy escasa estará indicada la anástomosis hipogloso o espino-ficial que, de ser necesario, podrá perfeccionarse con alguna forma de ciružría plástica. Si la secuela paralítica es menos importante pero constituye un serio inconveniente para el paciente, podrá considerarse alguna forma de intervención estética y funcional. En aquellos casos que presentan atrofia muscular total sin ninguna evidencia eléctrica de tejido muscular útil, sólo corresponderá recurrir a la cirugía plástica.

$\mathrm{Si}$, con la recuperación motriz, se presenta el síndrome de las lágrimas de cocrodilo (Bogorad) como en 6 casos sobre $58 \mathrm{~d} \epsilon$ James y Russell ${ }^{21}$, debido a que el facial ha sido lesionado a la altura o cerea del ganglio geniculado y a la mala dirección de las fibras secretoras regeneradas ${ }^{16,31}$ que, en vez de tomar el camino de la cuerda del timpano y del nervio lingual para llegar a las glándulas sublingual y submax:lar, lo hacen por el nervio petroso superficial mayor, estará indicada la secoión quirúrgica de este último nervio ${ }^{\top}$.

Psicoterapia - Así como, con igualdad de parálisis, la desfigıraciór del rostro varía según las diferentes fisionomías, siendo más marcada el. las de intensa gesticulación, rasgos más netos, arrugas más pronunciada: o piel más laxa, también con igual desfiguración facial la reacción an: mica del paciente varia según su personalidad psíquica, condicionada po. su genótipo $y$ las modificaciones paratípicas impuestas por el ambiente, la educación, la actividade social y la profesión. Por lo tanto, la artua. ción psicoterápica del médico variará según los enfermos. En realidad. el tratamiento directo de la parálisis con todos los recursos ya mencio- 
nados, bien prescriptos y aplicados, constituye una importante forma de psicoterapia indirecta.

La curación puede y debe ser siempre prometida pero sin especificar plazo y conviene explicar a la mayoría de los pacientes las distintas posibilidades evolutivas para todas las cuales hay tratamiento. Ante una incisiva exigencia de pronóstico exacto, éste deberá formularse con las lógicas reservas.

Ál enfermo se le deben señalar sus progresos para estimularlo a perseverar en la cura y disminuir su ansiedad. $Y$ los medios auxiliares a cargo del enfermo, aplicaciones locales de calor, inmovilización, ejercicios activos, deben indicarse siempre e insistir en su estricto cumplimiento. El enfermo que coadyuva en el tratamiento se solidariza con el médico en la prosècución de la cura y se torna mucho menos ansioso. Acepta más fácilmente, con más conformidad, una secuela, si llega a quedar, poique "sabe" que no ha colaborado, salvo rarísimas excepciones, con la detida asiduidad y entusiasmo en las indicaciones que se le han formulado. Halirá ido reduciendo el interés en la parte del tratamiento a su cargo a medida que disminuía su preocupación por la paresia o bien habrá disminuído su preocupación por la paresia a medida que, por desidia o cansancio, iba abandonando el tratamiento. En cualquiera de los dos casos, la carga afectiva del principio ha ido perdiendo fuerza lentamente para terminar por desaparecer.

Hemos visto algunos enfermos muy preocupados por su parálisis facial parcialmente mejorada, que permanecía estacionaria a pesar de la casi total normalización de las reacciones eléctricas que, al indicárseles ejercicios activos exclusivamente o asociados a masaje y galvanización, obtuvieron con rapidez apreciables mejorías estáticas y quinéticas al año y medio o dos años de la iniciación de la enfermedad y después de muchos meses de aparente estacionamiento, al mismo tiempo que la recuperación de un estado psíquico normal. Es indudable que en estos casos lo fundamental ha sido la reeducación motora y la acción personal del médico pues, a veces como acabamos de señalar, sólo habíamos indicado los ejercicios activos. Es muy posible que, en estos casos, la errónea dirección seguida por algunos axones regenerados y la ramificación de otros, han dificultado la recuperación funcional al haber creado nuevos modos de inervación periférica desadaptados de los engramas quinéticos previamente establecidos por la experiencia. Por eso, los movimientos activos, la reeducación quinética y la acción psicológica personal del médico son de tanta importancia y quizá permiten explicar ciertas rápidas curaciones o mejorías de paresias de larga data, atribuidas a intervenciones quirúrgicas tardías o a otros procedimientos.

\section{BIBLIOGRAFIA}

1. Allen, A. G.; Northíield, D. W. C. - Intra-oral splint for facial palsy. Lancet, 2:172, 1944. 2. Ballance, Ch. A.; Ballance, H. A.; Purves-Stewart, M. A. 
- Remarks on the operative treatment of chronic facial palsy of peripheral origin. Brit. M. J., 1:1009, 1903. 3. Ballance, Ch. A.. - () A casa of facial palsy treated by facio-hypoglossal anastomosis in which an anastomosis was also made between the spinal accessory and the distal segment of the divided hypoglossal nerve in order to prevent permanent lingual parlysis and atrophy. Lancet, $1: 1675,1909 ; b$ ) $A$ noe on the operative treatment of facial palsy with an account of some animal experiments. Brit. M. J., $1: 787,1932$. 4. Ballance, Ch. A.; Duel, A. B. - Operative treatment of facial palsy by introduction of nerve grafts into Fallopian canal and by other intratemporal methods. Arch. Otolaryng., 15:1, 1932. 5. Bauwens, Ph. i he electrodiagnostic aspect of facial paralysis. Proc. Roy. Soc. Med., 43:754, 1950. 6. Bierman, W. - Diagnosis and treatment of Bell's palsy. J.A.M.A., 149:253, 1952. 7. Boyer, F. C.; Gardner, W. J. - Paroxysmal lacrimation (syndrome of crocodile tears) and its surgical treatment. Arch. Neurol. a. Psychiat., 61:56, 1949. 8. Cwthorne, T. - Discussion on the limitations of operative treatment in traumatic facial paralysis. Proc. Roy. Soc. Med., 34:582, 1941. 9. Collier, D. J. - a) Discussion on the limitations of operative treatment in traumatic facial paralysis. Proc. Koy. Soc. Med., 34:575, 1941; b) Symposium: the treatment of facial paralysis. Proc. Roy. Soc. Med., 43:746, 1950. 10. Cooksey, F. S. - Discussion on the limitations of operative treatment in traumatic facial paralysis. Proc. Roy. Soc. Med., 34:580, 1941. 11. Delherm, L.; Laquerrière, A. - Electrología, vol. 21 del 'Irat. de Patol. Méd. y Terap. Aplic. Edit. Pubul, Barcelona, 1922. 12. DennyBrown, D.; Brenner, Ch, - Paralysis of nerve induced by ciirect pressure and by tourniquet. Arch. Neurol. a. Psychiat., 51:1, 1944. 13. Duel, A. B. - The operative treatment of facial palsy. Brit. M. J., 2:1027, 1934. 14. Eisenhauer, J.; Key, J. A. - Studies on muscle atrophy. A method of recording power in situ and observations on effect of position of immobilization on atrophy of disuse and denervation. Arch. Surg., 51:154, 1945. 15. Feinstein, B. - The application of electromyography to affections of the facial and the intrinsic laryngeal muscles. Proc. Roy. Soc. Med., 39:817, 1946. 16. Ford, F. K.; Woodhall, B. - Phenomena due to misdirection of regenerating fibers of cranial, spinal and autonomic nerves; clinical observations. Arch. Surg., 36:480, 1938. 17. Gutmann, E.; Gutmann, L. Effect of electrotherapy on denervated muscles in rabbits. Lancet, 1:169, 1942. 18. Hines, H. M. - Effects of immobilization and activity on neuromuscular regeneration. J.A.M.A., $120: 515,1942$. 19. Howe, H. A.; Tower, S. S.; Duel, A. B. - Facial tic in relation to injury of facial nerve; experimental study. Arch. Neurol. a. Psychiat., 38:1190, 1937. 20. Jackson, E. C. S.; Seddon, H. J. - InIluence of galvanic stimulation on muscle atrophy resulting from denervation. Brit. M. J., 2:485, 1945. 21. James, J. A.; Russell, W. R. - Bell's palsy: aetiology, clinical course and treatment. Lancet, 2:519, 1951. 22. Kinnier Wilson, S. A. Neurology. Edit. Arnold, London, 1:408, 1940. 23. Merwarth, H. R. - The occurrence of peripheral facial paralysis in hypertensive vascular disease. Ann. Int. Med., 17:298, 1942. 24. Monnier-Vinard; Puech, P. - Néphrite chronique et paralysie faciale. Bull. et Mém. Soc. Méd. d. Hôp. de Paris, pág. 977, 1930. 25. Moore, $\mathbf{E}$. W. - The fate of the face in poliomyelitis. Lancet, 1:1092, 1952. 26. Morris, W. M. - a) Surgical treatment of facial paralysis. Lancet, 2:1172, 1936; b) Surgical treatment of Bell's palsy. Lancet, 1:429, 1938. 27. Pickerill, H. P.; Pickerill, C. M. - Early treatment of Bell's palsy. Brit. M. J., 2:457, 1945. 28. Robbins, M. H. - Bell's palsy successfully treated with cortisone. Arch. of Ophth., 48:696, 1952. 29. Rothendler, H. H. - a) Bell's palsy treated with cortisone. J. Nerv. a. Ment. Dis., 114:346, 1951; b) Bell's palsy treated with cortisone. Am. J. M. Sc., 225:358, 1953. 30. Spillane, J. D. - Symposium: The treatment of facial paralysis. Proc. Roy. Soc. Med., 43:751, 1950. 31. Spiller, W. G. - Contiacture occurring in partial recovery from paralysis of facial nerve and other nerves. Arch. Neurol. a. Psychiat., 2:564, 1919. 